\title{
UN TERMINVS DE AGRIMENSOR DE CARÁCTER TÉCNICO PROCEDENTE DE LA SIERRA DE GATA (VILLAMIEL, CÁCERES)*
}

POR

\author{
ENRIQUE ARIÑO GIL
}

$\mathrm{Y}$

\author{
MIGUEL GARCIAA DE FIGUEROLA PANIAGUA \\ Universidad de Salamanca
}

\section{RESUMEN}

El trabajo que presentamos aborda el estudio de un epigrafe de fácil lectura pero de problemática intepretación procedente de Villamiel (Cáceres). El caracter rústico del documento no impide apreciar que en su labra se ha intentado acomodar el soporte a la forma de un terminus. Esto, unido a la indiscutible presencia de abreviaturas en las tres primeras lineas del texto, nos lleva a interpretar el epigrafe como un cipo vinculado a labores técnicas de agrimensura, destinado a ser leido por los mensores, quizá en relación con las operaciones de medicion del ager per extremitatem mensura comprehensus al que se refiere Frontino. Es también destacable la mención de dos comunidades desconocidas hasta ahora por las fuentes: Vinia (o Inia) Campegiensis y Valseni.

\section{SUMMARY}

The present work attends to the study of an epigraph from Villamiel (Cáceres) of easy reading but problematic interpretation. The rustic character of this document is not obstacle to appreciate the fact that in carving it, it was intended to accomodate the support to the form of a terminus. This, together with the evident presence of abreviations in the three first lines of the text, allows us to consider this inscription as a cippus related to technical labours of land-surveying, with the purpose of being read by the mensores, perhaps related with the measurement of the ager per extremitatem mensura comprehensus which Frontinus refers to. The mention of two communities, unknown until now, is also remarkable: Vinia (or Inia) Campegiensis and Valseni.

* El presente artículo es el primer resultado de la investigación que desarrollamos acerca del tema Implantación romana en el territorio de Salmantica, proyecto subvencionado por la DGICYT, con código PB 91-0419.

\section{INTRODUCCIÓN}

El artículo que presentamos tiene como objetivo el análisis de una inscripción poco usual. Este hecho, unido a las dificultades de interpretación que ofrece el documento, justifica sobradamente un tratamiento pormenorizado del epigrafe, pese a la brevedad del texto inscrito, seis líneas, por ende bastante parcas. Esta dificultad de interpretación viene marcada por la esencia del documento y no por dificultades de lectura, ya que el epigrafe nos ha llegado prácticamente completo y en excelente estado de conservación. Creemos además necesario hacer constar como advertencia preliminar que las conclusiones que aqui desarrollamos nos parecen las más ciertas, dentro de unas posibilidades reducidas, pero sería faltar a la honradez el presentarlas como absolutamente seguras'.

\footnotetext{
'Queremos agradecer aquí la amabilidad de los doctores José Antonio Abásolo, Josep Maria Gurt y José Luis Ramirez Sádaba, con quienes intercambiamos puntos de vista a propósito de la interpretación del epigrafe. Huelga decir que somos los únicos responsables de cuanto se expresa en el texto que presentamos a continuación. También estamos en deuda con David Ariño, quien proporcionó asesoramiento sobre la coherencia sintáctica de la restitución que damos al texto, especialmente en lo que se refiere a las abreviaturas de las tres primeras lineas.
} 


\section{ANTECEDENTES Y CIRCUNSTANCIAS DEL HALLAZGO}

La comarca de la Sierra de Gata se sitúa en el ángulo Noroeste de la provincia de Cáceres. limitando al norte con Salamanca y al oeste con Portugal. Geológicamente esta zona forma, junto con la Sierra de Francia, un segmento del Sistema Central, definido a un lado por el Corredor de Béjar y al otro por el de Las Mesas, que le separa de las prolongaciones portuguesas de la Sierra del Gardunho y de la Estrella. sion. distribuidos por laderas y valles y que nos hablan de la existencia de un hábitat disperso semejante al conocido en la zona hasta hace bien pocos aǹos 2 . Las mismas prospecciones han permitido mostrar también la existencia de núcleos de población importantes situados en las zonas más bajas, en el limite con la penillanura extremeña. Muchos de ellos parecen ser asentamientos antiguos que podrian haber entrado en contacto con la cultura romana sin ningún tipo de traumas. Asi parece ocurrir con el asentamiento de "Val de los Pozos», en el tér-

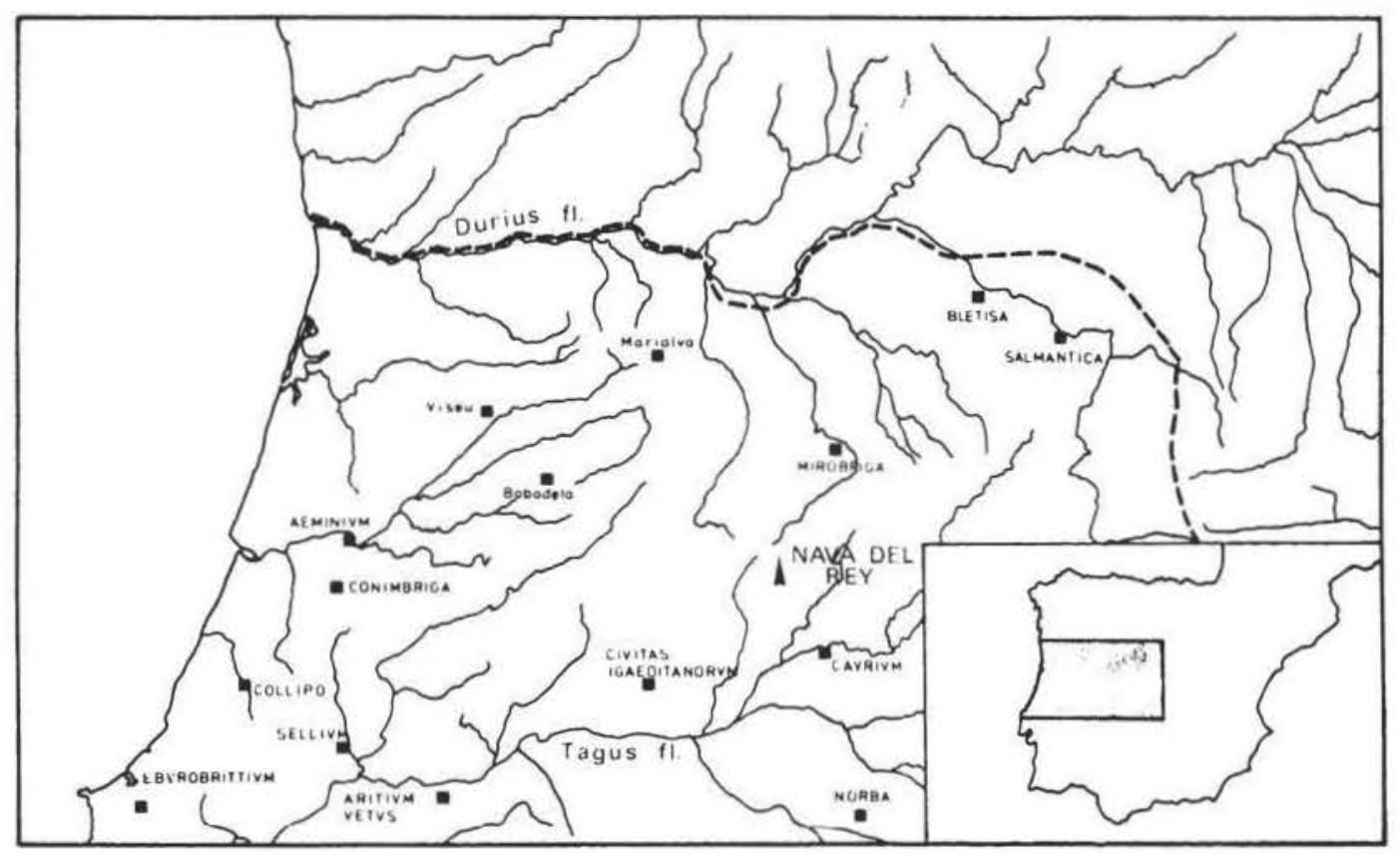

Figura 1. Localización del epigrafe en relación con el entorno urbano.

Lo que conocemos del poblamiento durante la época romana es realmente poco. Situada en una zona de dificiles comunicaciones, las ciudades conocidas más próximas a la comarca fueron Mirobriga (Ciudad Rodrigo) al norte, Caurium (Coria) al sur y la ciuitas Igaeditanorum (Idanha-a-Velha) al este, sin que podamos establecer por el momento el tipo de relación que los habitantes de la sierra tuvieron con ellas. Las prospecciones realizadas desde los años ochenta han permitido localizar más de cincuenta yacimientos, en su mayoría de pequeña exten- mino de Villamiel, uno de los núcleos con mayor entidad durante la época romana, a la vista de su extensión y de los materiales estudiados ${ }^{3}$. En relación de proximidad con "Val de los Pozos» se conocen otros yacimientos, como el de "Los Trechados», "Villalba" o "La Nava

: Garcia-Figuerola, Miguel: Introducción a la carta arqueológica de la Sierra de Gata, Memoria de Licenciatura inédita. Salamanca, 1983.

Ver por ejemplo Torres Peralta, Maria Jesús: Estelas inéditas en la provincia de Cáceres. Homenaje al profesor Martin Almagro Basch, Madrid, 1983, 365-369. 
del Reyn ${ }^{4}$, en el mismo término municipal, siendo este último el lugar de donde procede el epigrafe que aqui presentamos.

La inseripción fue localizada hace algunos años formando parte de una cerca, junto al caserio ${ }^{\varsigma}$. La información recibida entonces parece situar el lugar original de procedencia en sus inmediaciones, bien en el mismo teso, bien en el contiguo, entre las ruinas de la ermita de Nuestra Señora de la Oliva. Hoy en dia se encuentra en San Martin de Trevejo, en casa de D. Luis Bacas, a quien agradecemos las facilidades que hemos encontrado para su estuciio.

El epigrafe habia sido publicado por uno de nosotros " junto a otras inscripciones de la zona. La singularidad del documento nos ha tentado a volver sobre su estudio, con la esperanza de desentrañar su significado.

\section{DESCRIPCIÓN Y ESTADO DE CONSERVACIÓN}

La pieza que aquí presentamos corresponde, como se verá, a un terminus elaborado en pizarra. El soporte apenas ha sido trabajado por el marmorarius, que se ha limitado a aprovechar la forma sensiblemente piramidal de la roca, tallando y puliendo exclusivamente la parte destinada a acoger al texto. El cipo presenta un somero trabajo en su parte superior que lo configura casi a modo de fastigium. En su parte derecha superior la inscripción ha sufrido una rotura que apenas afecta parcialmente a la primera línea. Por lo demás, el documento está prácticamente completo y sólo presenta un desconchado a la altura del principio de la tercera línea, que afecta solamente a la letra inicial. Las dimensiones de la pieza varían en virtud del punto en que se tome la medida, dado el carácter tosco que presenta el epígrafe. La al-

\footnotetext{
4 Melena, José Luis: Salamanca, Jalama y la epigrafia latina del antiguo Corregimiento, Symbolae, Ludouico Mitxelena septuagenario oblatae, Salamanca, 1985, 475530 .

'Las coordenadas son $40^{\circ} 08^{\prime} 45^{\prime \prime}$ de latitud y $3^{\circ} 09^{\prime}$ $30^{\prime \prime}$ de longitud $\mathrm{W}$ respecto al meridiano de Madrid.

- Garcia-Figuerola, Miguel: Inscripciones romanas de la Sierra de Gata, Alcántara, Mayo-Agosto, 1989, 131132, foto 7 .
}

tura máxima es de $0,79 \mathrm{~m}$, con un ancho máximo de $0.56 \mathrm{~m}$ y un espesor de $0,40 \mathrm{~m}$. El campo epigráfico tiene $0,68 \mathrm{~m}$ de alto por $0,44 \mathrm{de}$ ancho. La altura de las letras oscila entre los 3,5 y $\operatorname{los} 9 \mathrm{~cm}$. La distancia entre líneas varia entre $1 \mathrm{~cm}$ y $6 \mathrm{~cm}$ siendo destacable que el alineamiento no es perfecto por lo que se producen variaciones en una misma línea.

El texto es perfectamente legible y sólo la primera letra de la tercera linea se encuentra algo deteriorada, tal como acabamos de decir, pese a lo cual parece segura la lectura de esta letra como una $\mathrm{V}$, menos probablemente una $\mathrm{N}$. Como particularidades paleográficas destacar únicamente que la última letra de la primera línea, una $\mathrm{M}$, se adapta al marco, deformándose a consecuencia de la falta de espacio con que el grabador tuvo que enfrentarse a causa de una mala ordinatio. La quinta letra de la tercera linea presenta una factura deficiente a consecuencia de una fisura natural de la roca. Las dos primeras letras de la segunda línea están unidas por un nexo y la lectura puede ser tanto TE como ET.

\section{ANÁLISIS DEL EPÍGRAFE}

Antes de empezar una interpretación del texto que el monumento epigráfico nos transmite queremos insistir, como ya haciamos al principio del trabajo, en el hecho de que es difícil establecer un análisis seguro, dado lo abreviado de la inscripción. Nuestro objetivo va a ser el de intentar comprender la función del documento a partir de los datos que consideramos, en principio, más ciertos o al menos más probables. A partir de ellos intentaremos interpretar las partes del epigrafe más oscuras. La parquedad del texto inscrito nos ha hecho prestar especial atención a un capítulo que, imprescindible en todos los estudios epigráficos, reviste en nuestro caso una especial importancia. Nos estamos refiriendo al análisis del soporte y la paleografía.

Como punto de partida pensamos que hay que asumir una serie de hechos como altamente probables. En primer lugar creemos que existe una absoluta coherencia en el texto inscrito. Lo 


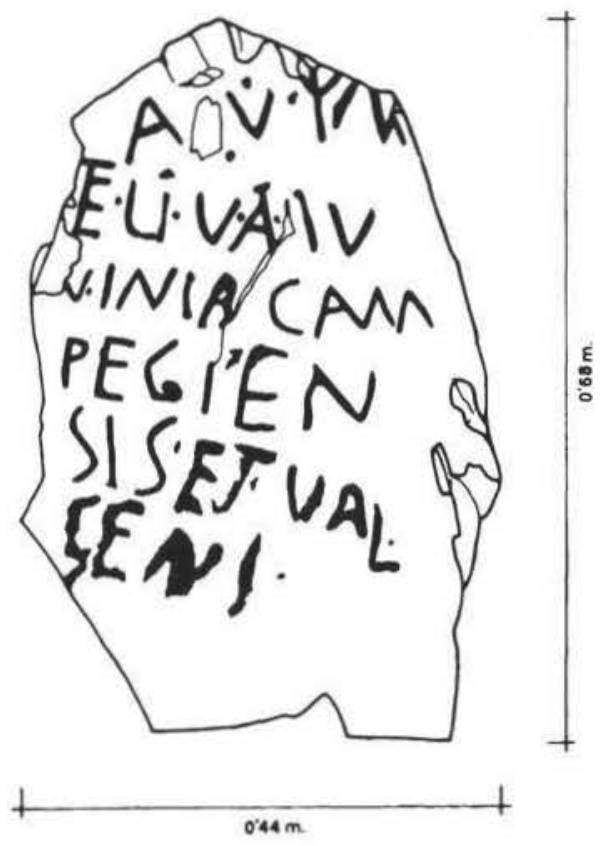

Figura 2.-Dibujo del epigrafe, realizado partiendo de una reducción del calco original.

cual permite afirmar que o bien el lapidarius entendia perfectamente el texto, o bien existió un control directo por parte de la persona que lo encargaba. En cualquier caso el hecho fue que los puntos de separación se situaron de manera lógica y precisa de modo que facilitaban la comprensión del texto pese a su brevedad. En este mismo sentido podemos pensar que el soporte fue elegido en relación con el texto. Pese a su apariencia rústica, el soporte ha sido configurado para acomodarse a la función deseada. Así en su extremo superior se ha procedido a tallarlo, retocando con este fin el bloque que, ya por su configuración natural, asemejaba el aspecto deseado de un terminus. $\mathrm{El}$ somero trabajo preparatorio al que ha sido sometido el bloque natural de piedra no ha impedido que el campo epigráfico haya sido cuidadosamente alisado. Esta circunstancia, junto a la diferencia en la técnica de inscripción que se observa, nos ha llevado a determinar que las dos últimas palabras (et Valseni) se añadieron con posterioridad a haberse fijado el monumento sobre el terreno. En efecto, la parte inferior del soporte, donde se sitúan estas últimas palabras, no ha sido preparada y alisada como el resto del campo. Por otro lado, hasta la mitad de la quinta linea incluida (sis) el scalprum se ha utilizado para configurar las letras en la habitual forma de surco $7 \mathrm{y}$ a partir de ahi las letras se han inscrito por medio de un piqueteado a base de puntos.

Otra idea de la que hemos partido para nuestra interpretación, ha sido la convicción de que el uso de las abreviaturas se justifica por la naturaleza técnica del documento, perfectamente inteligible dentro de un contexto destinado para un personal especializado y familiarizado con él. Aunque con menor grado de seguridad, y atendiendo a la coherencia del documento, pensamos que puede afirmarse que cada una de las tres primeras lineas, donde se concentran las abreviaturas, expresa por sí misma una idea o ideas completas. Igualmente, $y$ asumiendo este hecho, creemos que existe un orden de importancia en la exposición de estas ideas de tal manera que lo que se quiere destacar figura en la primera linea.

A continuación procederemos a un análisis pormenorizado de cada una de las líneas con el fin de poder aportar una interpretación de conjunto.

$$
\text { Linea } 1: \mathrm{A} \cdot \dot{\mathrm{V}} \cdot \mathrm{PM}
$$

Partiendo del lugar que ocupan los puntos de separación podemos afirmar que contamos en esta línea con tres significantes distintos. Las claves de interpretación se basan en la lectura de la $\mathrm{V}$ como numeral y de PM como passuum milia. La identificación de la $\mathrm{V}$ como numeral se basa tanto en el punto que figura encima de la letra como en la coherencia que esto presenta con la lectura de PM como passuum milia. Sobre esta interpretación para las dos últimas letras de la línea hay que decir que, si bien la abreviatura más usual es MP, la abreviación en PM no carece de ejemplos documentados ${ }^{8}$. Par-

Utilizamos la terminología de Di Stefano Manzella. Ivan: Mestiere di epigrafista. Guida alla schedatura del materiale epigrafico lapideo, Roma, 1987, 138-139.

${ }^{x}$ La abreviatura PM aparece por ejemplo en un miliario procedente de Pozalmuro (Soria), CIL, II, 4896. Cfr. Mañanes, Tomás; Solana Sainz, José Maria: Ciudades y vias romanas en la cuenca del Duero (Castilla-León). Va- 
ticndo de ambos supuestos que nos parecen altamente probables. consideramos la $\mathrm{A}$ inicial como una preposicion que introduce el numeral.

Linea 2: TE $\cdot$ LI $\cdot V \cdot A \cdot I V$

Los puntos de separación nos marcan aqui cinco palabras distintas. El primero de ellos presenta en nexo las letras T y L. Pensamos que la lectura debe ser te(rmimus), en coherencia con la siguiente palabra que abreviada en I.I hay que leer como li(mitis) o, quiza. li(mitalis)". El resto de la linea es de más dificil interpretación pero cabe suponer aqui la presencia de dos numerales separados por una preposición. La V (quintus) la hemos relacionado con terminus y nos daria la numeración del mojón. La preposicion A remitiria al punto IV (el terminus quarIus ) desde el que se mide la distancia que figura en la primera linea.

Lineas 3, 4. 5 y 6: VINIA CAM . / PEGIEN/SIS - ET . VAL • / SENI

La dificultad principal de esta parte radica en la linea tercera y consiste en desentrañar si existe punto de separación entre la primera letra (una $\mathrm{V}$, menos probablemente una $\mathrm{N}$ ) y la segunda (una 1). La marca es muy débil y no podemos asegurar si se trata de algo intencionado o es resultado de un piqueteado accidental, semejante al que aparece en otras partes de la inscripcion. La separación entre ambas le-

lladolid. 1985, $n * 33,157-158$. También en dos miliarios procedentes de la provincia de Orense, uno de ellos de la zona de Portela d'Home y el otro de Bollo (CIL. II, 4843. Rodriguez Colmenero. Antonio: La red viaria romana del sudeste de Galicia, Valladolid, 1976, n." 9, 105: n." 30. 108). A titulo de ejemplo fuera de Hispania podemos citar su presencia en miliarios africanos: CII. X, 22340; 22370. En un cipo de delimitación de campos privados (CIL. VI. 29775 ) figuran de forma expresa las palabras passus mille. En CIL. VI. 29778 se lee igualmente passu(s) mille.

- El término más usual, tanto en general como referido a labores de agrimensura, es limes, pero al sentido del epigrafe se adapta mejor, en nuestra opinión, limitalis. Cfr. sendas voces en Thesaurus Linguae Latinae, volumen VII. pars altera, sectio II, Leipzig. 1970-1979. Especialmente significativa nos parece la expresión fossae limitales que se encuentra en la Lex Vrsonensis, 104, II, apud. D'Ors: Epigrafia juridica de la España romana. Madrid, 1953, 236. trats no es significativa y podriamos cstar ante una unica palabra. Los unicos datos para establecer el sentido de esta parte está en las pala. bras siguientes, Campegiensis el lalseni. que parecen aludir a unas comunidades. Fl caso en que se encuentran estas dos localidades es dificil de determinar, siendo necesario elegir entre nominativo singular o genitivo singular para Campegiensis y nominativo plural o genitivo singular para I alseni. palabra coordinada a la anterior por la conjunción et. La coherencia de la interpretación global obliga a leerlas en nominativo e identificarlas con las comunidades implicadas en las operaciones técnicas que se indican en el termimus.

La duda acerca de la primera o primeras palabras de la tercera linea subsiste, pero pensamos que, teniendo en cuenta que el étnico Campegiensis parece adjetivarla es probable que nos encontremos con el nombre de una población en nominativo.

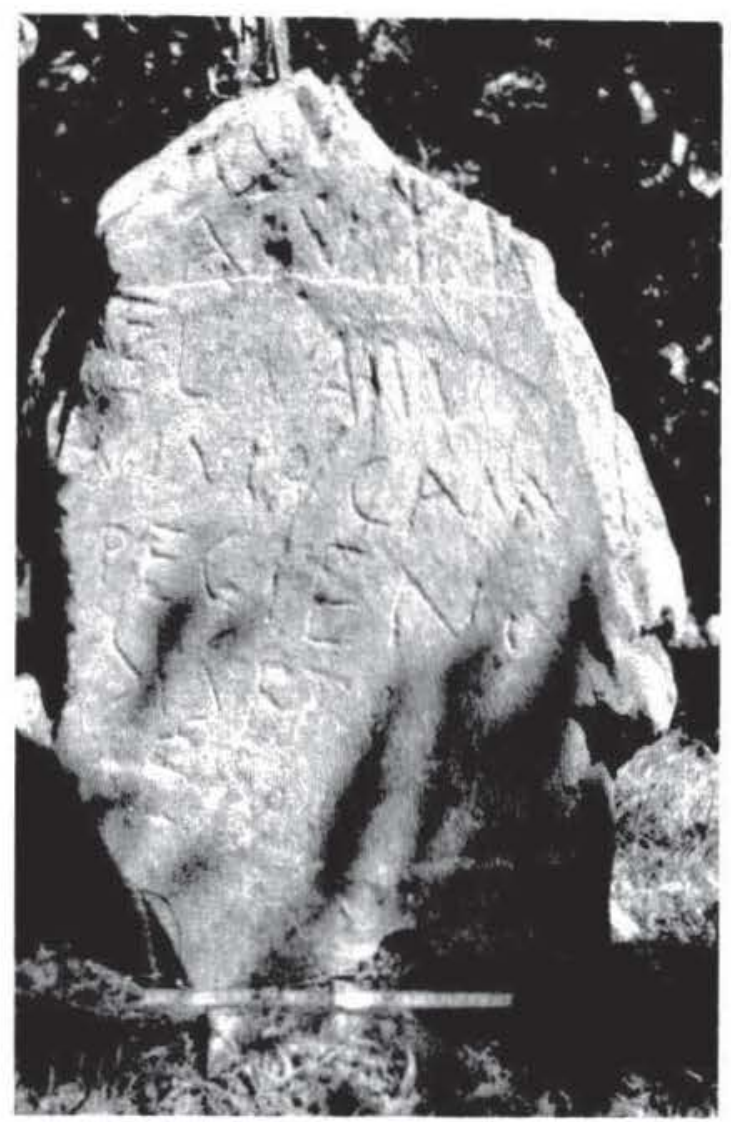

Figura 3.-Fotografia de la inscripcion. 


\section{CONCLUSIONES E INTERPRETACIÓN}

A la vista de los datos expuestos en el apartado anterior nuestra transcripción, aún con las lógicas reservas ante la brevedad del texto, es la siguiente:

A(nte) $V$ p(assuum) m(ilia). / Te(rmimus) li(mitis) V a(nte) IV. / Vinia Cam / pegien /'sis et Val/seni.

Creemos, en consecuencia, que estamos ante un epigrafe técnico, relacionado muy probablemente con las labores de una limitatio o depalatio y que cobra su pleno sentido dentro de este contexto y en relación con otras piezas semejantes. En nuestra opinión estamos ante un documento de carácter interno, es decir, instalado por mensores como referencia para las operaciones de medida. Esto justifica el uso de un lenguaje abreviado ${ }^{10}$, la importancia de los numerales, la ausencia de verbos, la forma esquemática en que figuran las comunidades al final de la inscripción asi como la rusticidad del soporte y la grafia.

\footnotetext{
10 Es propio del lenguaje técnico de los agrimensores este carácter abreviado. Aunque carecemos de paralelos en sentido estricto para la inscripción que presentamos puede verse el uso de abreviaturas como norma en documentos como los catastros en mármol de Arausio (Orange): Piganiol, André: Les documents cadastraux de la colonie romaine d'Orange. Paris, 1962. En lo que a termini de división de tierras se refiere, el uso de abreviaturas era constante y asi lo hace constar el Corpus Agrimensorum Romanorum, especialmente el tratado De terminibus. Esto está atestiguado tambièn por los epigrafes en un número tan abundante que huelga citar ejemplos aqui. De creer a los agrimensores existian incluso unas claves de lectura para simples marcas en los cipos, que indicaban, al que estaba familizarizado con ellas, la existencia de una fuente, un arroyo, un pozo, un depósito de agua o una línea de árboles por ejemplo. A propósito de ello pueden encontrarse referencias en Dilke, O.A.W.: The Roman Land Surveyors. An Introduction to the Agrimensores, Newton Abbot, 1971, 98-105. El uso de abreviaturas en los cipos de limitationes es usual en todos los casos documentados, si bien éstas son muy uniformes y por ello de comprensión más fácil y directa que las que presenta nuestro terminus. Vid. un repertorio bibliográfico al respecto en Hinrichs, Focke Tannen: Die Geschichte der gromatischen Institutionen. Untersuchungen zu Landverteilung. Landvermessung. Bodenverwaltung und Bodenrecht im römischen Reich, Wiesbaden, 1974 , nota 45,58 . Ver también la traducción
}

Entre la información que el texto transmite destacan tres aspectos distintos. En primer lugar hay que señalar que la inscripción comienza con la distancia respecto a un punto anterior medida en passuum milia. Este debió ser el aspecto más importante para el trabajo que se estaba realizando. En segundo lugar figura la numeración del terminus y su relación con el precedente, respecto al cual se mide dicha distancia. En tercer y último lugar se hace constar los colectivos cuyas tierras son objeto de medición. En nuestra opinión se midió en primer lugar el perimetro de la comunidad de ¿Vinia? Campegiensis, siendo después utilizado el mismo terminus para el perimetro de la comunidad colindante de los Valseni, cuyo nombre se añade con posterioridad ".

Aunque no conocemos paralelos idénticos al epigrafe que analizamos, el CIL, VI, 29793 presenta una distancia medida en pies entre dos cipos que se numeran formando parte de una serie y que parecen corresponder a los limites de una pequeña propiedad ${ }^{12}$. Sabemos además que este tipo de operaciones eran frecuentes en los trabajos de agrimensura. Por ejemplo CIL. $\mathrm{VI}, 1268^{13}$ se refiere a una depalatio que con-

francesa de esta obra: Histoire des institutions gromatiques, Paris, 1989, nota 45, 60. También Favory, François: Propositions pour une modélisation des cadastres ruraux antiques, Cadastres et espace rural. Approches et réalités antiques, Paris, 1983, nota 9. Vjemplares más recientes de cippi gromatici han aparecido en Mantua (Roffia, Flisabetta: Il cippo gromatico rinvenuto a Mantova in piazza Sordello, en Misurare la terra: centuriazione e coloni nel mondo romano. Il caso mantovano, Catálogo de Exposición de Mantua, 25 de febrero - I de abril de 1984, Módena, 1984, 82-86) y en Campania (Chouquer, Gérard: Les éléments d'un paysage intégré. Le tissu rural, en Chouquer, Gérard: Clavel-Lévêque, Monique; Favory, François; Vallat, Jean Pierre: Structures agraires en Italie centro-méridionale. Cadastres et paysages ruraux, Roma, 1987. 308-313). Un buen numero de ellos han aparecido vinculados a las centuriaciones del norte de Africa: Trousset, Pol: Les bornes du Bled Segui. Nouveaux aperçus sur la centuriation romaine du Sud Tunisien, Ant. Afr., 12, 1978, 125-177.

"Aunque en la restitución que proporcionamos del texto hemos optado por leer Vinia como primera palabra de la cuarta linea, hay que valorar la posibilidad de que el nombre de la comunidad fuese Inia, siendo la primera letra de intepretación más problemática.

12 Cippus dec(i)mus. A cippo / dec(i)mo ad primum ped(es) / LXXXXVII.

13 Hi termini XIX positi sunt / ab Scriboniano et Pisone Frugi / ex depalatione T(itii) Flaui Vespasiani / arbitri. 
Lleva la numeración de los termini y lo mismo ocurre en CIL. VIII, $25366^{14}$. También se documentan cippi sin numeración pero con referencias topográficas utilizadas para la delimitación de campos privados ${ }^{15}$. La actio finium regundorum implica operaciones semejantes pues el establecimiento del finis supone describir su recorrido y marcarlo con cipos correlativos ${ }^{10}$.

No estamos en condiciones de afirmar cuál fue la causa que llevó a establecer la medición del perimetro de las tierras de las comunidades citadas, si se debió a una iniciativa particular o estatal, asi como tampoco podemos afirmar si existió o no un litigio de tierras como transfondo - aunque esto nos parece menos probableya que el epigrafe no lo indica de ninguna manera. Sí que nos parece pertinente resaltar que la tierra parece haber sido medida por la totalidad de su perimetro, dada la amplitud de la medida expresada en el terminus, lo que resulta tentador relacionar con el ager per extremitatem mensura comprehensus que menciona Frontino ${ }^{17}$. Aunque este tipo de ager fue sin duda algo muy extendido en todo el Imperio, es destacable que uno de los dos ejemplos que cita este gromaticus es justamente el de la ciudad de Salmantica en Lusitania. Este sistema de medición de campos es mal conocido pero sabemos que se define midiendo la totalidad de su superficie partiendo de su perímetro. Frontino especifica que es el sistema de organización territorial propio del suelo tributario en las provincias, por lo que cabe asociarlo a las ciudades estipendiarias. En el ager per extremitatem mensura comprehensus el estado romano se limitaría a establecer la superficie total de tierras de una ciudad fijando el impuesto global a las autoridades de la misma según el re-

\footnotetext{
14 TERM N N VII / T ANNI / AMPLIATI ab allera parte: TERM N VIII/ IVLIORVM FLACCI ET CHLLS. También CIL, VIII, 25988 presenta un conjunto de cippi con numeración pertenecientes a la ciudad de Thugga.

is Por ejemplo CIL, VI, 29771; 29772; 29782.

16 Sobre la actio finium regundorum y los epigrafes que a ella se refieren vid. Hinrichs, Focke Tannen: Die Geschichte der gromatischen ..., 171-223, cit. (n. 10); Histoire des institutions... 181-234, cit. (n. 10).

17 Frontino, Th, 1-2.
}

sultado, quedando la recaudación bajo la responsabilidad de la administración urbana ${ }^{1 x}$. El epigrafe que aqui presentamos podria ser un ejemplo no sólo de este tipo de campo, sino del sistema que se utilizaba para su medición, y testimoniaria el uso de esta técnica para las colectividades.

Otra cuestión espinosa a la que hay que aludir es la que se refiere a la identificación de las localidades que el terminus recoge. A este respecto poco podemos aportar puesto que ninguna de las dos es conocida, al no ser mencionada ni por las fuentes ni por la epigrafia ${ }^{19}$. La identificación de las localidades con yacimientos concretos es muy difícil aunque a titulo de hipótesis queremos mencionar la posibilidad de que el yacimiento de "Val de los Pozos» (Villamiel, Cáceres), muy cercano al lugar del hallazgo, se corresponda con una de las dos.

Por último es necesario aludir a la cronología de la pieza, tema sobre el que no podemos pronunciarnos puesto que el epígrafe carece de criterios internos de datación y tampoco tenemos datos proporcionados por un contexto arqueológico. La paleografia, muy condicionada por el material del soporte y el carácter rústico del documento, no permite extraer ninguna conclusión. Si analizamos el epígrafe desde el punto de vista de un marco histórico general y lo in-

1* Hinrichs, Focke Tannen: Die Geschichte der gromatischen..., 114-115, cit. (n. 10); Histoire des institutions.... 120-121, cit. (n. 10). Vid. taınbièn Salinas de Frias, M.: Sobre las formas de propiedad comunal en la cuenca de Duero en época prerromana, Veleia, 6, 1989, 103-110.

${ }^{19}$ No obstante es necesario aludir a los termini de delimitación entre Mirobriga (Ciudad Rodrigo) y Salmantica (Salamanca) (CIL, II, 857) y Mirobriga y Bletissa (Ledesma) $(C I L, I I, 858)$, donde el nombre de las localidades se menciona seguido de VAL, abreviatura de dificil interpretación. Las inscripciones están hoy perdidas pero tanto Cabañas como Ponz, los copistas que las transmiten, coinciden en este punto. Puede encontrarse un estado de la cuestión sobre el problema del significado de VAL, asi como sobre el problema de la exacta procedencia de los termini, en Mangas, Julio: Ciudades antiguas de la Provincia de Salamanca (siglo III a.C. - Diocleciano), Actas del I Congreso de Historia de Salumanca, vol. I, Salamanca, 1992, 251-268. No obstante, valorando todos los datos que tenemos, pensamos que no es seguro identificar la abreviatura con una comunidad ni, por lo tanto, nos parece legítimo relacionarlo con los Valseni de la inscripción que aqui estudiamos. 
terpretamos como correspondiente a las operaciones técnicas de delimitación del ager per extremitatem mensura comprehensus, lo que nos parece lo más probable, sería posible fecharlo incluso en época augustea ${ }^{20}$, fecha que parece excesivamente temprana para la zona. No nos parece descartable una datación en épo-

20 Focke Tannen Hinrichs: Die Geschichte der gromatischen..., 115, cit. (n." 10): Histoire des institutions..., 121. cit. $(\mathrm{n}, " 10)$, piensa que la medición del ager per extremitatem mensura comprehensus en Lusitania fue iniciativa de Augusto y simultánea de las operaciones de asignación de tierras en Augusta Emerita, en conexión con la reestructuración de la nueva provincia. Es de destacar que los ca flavia en relación con la politica de revisión del catastro llevada a cabo por Vespasiano ${ }^{21}$. Al respecto queremos destacar que la pobreza y mala factura del monumento epigráfico no debe llevarnos a presuponer necesariamente una datación tardia, por otra parte no descartable.

termini CIL, II, 857,858 y 859 que se refieren a los territorios de Bletissa. Mirohriga y Salmantica llevan datación del año $6 \mathrm{~d}$. C

2 Sobre la politica de revisión del catastro llevada a cabo por Vespasiano vid. Hinrichs, Focke Tannen: Die Ges chichte der gromatischen..., 128-146, cil. (n. $\left.{ }^{\circ} 10\right)$ : Histoire des institutions.., 135-154, cit. (n. $\left.{ }^{\circ} 10\right)$.

\title{
LOS MOSAICOS COMO DOCUMENTOS PARA LA HISTORIA DE LA HISPANIA TARDÍA (SIGLOS IV-V)
}

\author{
POR \\ JAVIER ARCE \\ Escuela Española de Historia y Arqueología del C.S.1.C. - Roma
}

RESUMEN

El presente artículo examina algunos ejemplos de mosaicos relevantes hallados en contextos arqueológicos de la Hispania tardorromana (Iv $-\mathrm{v}$ d. C.) e investiga su validez como "fuente para la Historia tardorromana». Recientes investigaciones $\mathrm{e}$ interpretaciones han pretendido verlos como una prueba de religiosidad de los propietarios de las villae en las que fueron encontrados.

Mi propuesta es que han sido "superinterpretados" y que es necesario ser muy cautos cuando se realizan dichos estudios, teniendo en cuenta el contexto histórico de la producción musiva del período tardorromano.

\section{SUMMARY}

The present article examines some examples of relevant mosaics founded in archaeological context of Late Roman Spain (IV-v A. D.) and investigates their validity as "source for the Late Roman History». Recent research and interpretations of them claim their meaning as a proof of religious commitments for their owners or even ownership of the villae in which were found.

The proposal is that they have been overinterpreted and that it is necessary to be very cautious in its study taking into account the historical background of mosaic production of the Late Roman period. 